\title{
La organización de la Investigación en Atención Primaria
}

\begin{abstract}
La Atención Primaria, como eslabón esencial del sistema sanitario, tiene un área de conocimiento que le es propia y que debe actualizar y renovar permanentemente. Se relaciona ampliamente con otras áreas de conocimiento y se nutre de diferentes disciplinas y métodos, tanto provenientes de las ciencias de la salud como de las ciencias sociales, pero aportando una visión y perspectiva diferente para responder más específicamente a sus necesidades. Así, en la medida en que la Atención Primaria (AP) sea capaz de generar nuevo conocimiento, de responder a la falta de evidencia existente en las decisiones que se $\operatorname{toman}^{1}$ y de mejorar la calidad de sus servicios y la salud de los ciudadanos, estará creando una investigación propia y útil para la $\mathrm{AP}^{2,3}$.
\end{abstract}

La investigación se basa en el talento científico de los investigadores y grupos para hacerse preguntas relevantes y obtener respuestas válidas. Pero su desarrollo es cada vez es más complejo y competitivo y tiene unas duras reglas de juego que hay que asumir. Y para ello es imprescindible conseguir recursos y tener el apoyo de una buena organización. Veamos pues como debería organizarse la investigación en AP.

1.Investigadores: no todos los profesionales deben investigar, y no todos los que quieren investigar deben estar en "primera división". Pero es imprescindible que haya una investigación de excelencia si queremos una AP de calidad, con investigadores bien formados dedicados parcialmente o totalmente a la investigación.

2.Grupos: la unidad básica de la organización de la investigación son los grupos de investigadores que desarrollan líneas de investigación estables a partir de la realización de proyectos multicéntricos. Es imprescindible que los investigadores del grupo se centren en pocas líneas de investigación para poder profundizar en sus contenidos y necesidades de conocimiento y obtener una sólida experiencia. Además es ne- cesario salir del propio centro de atención primaria y de la investigación local para trabajar con otros centros de AP o con otras especialidades relacionadas, formando grupos multicéntricos e interdisciplinares de investigación.

3.Instituciones: el grupo de investigación necesita tener una organización que le proporcione una serie de servicios, sin los cuales difícilmente podrá sobrevivir: para el desarrollo de los proyectos necesitará profesionales o unidades de soporte cada vez más especializadas (procesamiento de datos, estadística, informática, soporte en metodología cualitativa, etc.); el proyecto deberá estar aprobado por un Comité de Ética e Investigación Clínica (CEIC); el grupo deberá estar informado de las distintas convocatorias de ayuda, tendrá que ser capaz de gestionar los recursos económicos y humanos de sus proyecto y del grupo, y las memorias anuales y finales; también deberá tener el soporte de las instituciones involucradas para facilitar el trabajo de los investigadores y su dedicación al proyecto, etc. A su vez, estas organizaciones deben ser capaces de responder a los retos cada vez más complejos y competitivos de la investigación: participación en proyectos internacionales, acreditación de grupos de excelencia en el ámbito nacional o europeo; mayor dedicación y profesionalización de los investigadores, creación de plataformas especializadas de soporte a la investigación (epidemiología y estadística, mantenimiento de cohortes, informática, laboratorios especializados, elaboración y control de grandes bases de datos, etc.); participación en redes nacionales e internacionales; seguimiento de las buenas prácticas en investigación; gestión de los recursos (becarios, investigadores, equipamientos, contabilidad, etc.); búsqueda de financiación y sostenibilidad.

Por tanto, es imprescindible que la AP organice su investigación, y al igual que lo hace con la asistencia y la docencia, debe crear orga- 
nizaciones especializadas que faciliten estos servicios e infraestructuras de soporte a los grupos y establezcan estrategias que permitan consolidar sus líneas de investigación, teniendo en cuenta las políticas de la comunidad autónoma, estatales e internacionales.

4.Redes: a su vez, estos grupos y organizaciones deben intentar participar en infraestructuras más amplias de investigación en las que poder encontrar colaboración y complementariedad al desarrollo de sus líneas de investigación: redes nacionales (por ejemplo, el programa RETICS del Instituto de Salud Carlos III) e internacionales, consorcios europeos, etc. La existencia de la redIAPP (Red de Investigación en Actividades Preventivas en Atención Primaria) es un buen ejemplo y modelo de organización de una red de grupos de investigación de AP en España (www.rediapp.org) ${ }^{4}$. Sin embargo, esta dimensión nacional e internacional de la investigación en AP aún está en sus inicios y es uno de sus grandes retos de futuro, dependiendo en gran manera del futuro desarrollo de organizaciones sólidas de investigación en AP.

En la creación de estas organizaciones de investigación deben tenerse en cuenta una serie de elementos fundamentales:

1.La investigación debe realizarse desde instituciones independientes, Institutos o Centros de Investigación. Deben ser instituciones especializadas en investigación, con gran agilidad en la gestión de recursos económicos y humanos, y capaces de desarrollar estrategias propias de investigación ligadas a las políticas de $|+D+|$ más generales. La contratación de los investigadores no puede estar sujeta a los criterios de contratación de los profesionales asistenciales ni de los presupuestos anuales cerrados. Además, la contratación debe ser ágil, debe tener unos criterios de baremación específicos en función de las tareas de investigación que desarrollan y se requiere flexibilidad tanto en la duración como en la intensidad de los contratos. Todo ello hace muy difícil la adaptación a los contratos asistenciales.

2.Asimismo deben estar íntimamente relacionadas con las instituciones de provisión de servicios de la comunidad, ya que deberán trabajar conjuntamente con sus profesionales, pero con una gestión independiente.

3.Deben ser instituciones que abarquen amplios territorios (como por ejemplo toda una comunidad autónoma). La AP está muy dispersa en centros pequeños y para evitar una investigación localista es necesario coordinar amplios territorios en los que se puedan formar distintos grupos de investigación y realizar proyectos multicéntricos. Es muy importante evitar la fragmentación de la AP (en ámbitos pequeños, zona de influencia de un hospital, etc.).

4.A diferencia de la investigación de un hospital, la organización debe ser descentralizada, creando unidades de soporte territorial próximas a los profesionales, que promuevan y dinamicen la investigación en el territorio coordinando investigadores y formando equipos y grupos estables de investigación, que asesoren los proyectos y que den soporte a su ejecución. Estas unidades deben estar vinculadas a las entidades proveedoras de servicios de AP de su territorio (para promover estrategias específicas de desarrollo de la investigación) y coordinadas con las otras unidades de soporte de la comunidad autónoma para fortalecer las estrategias de investigación en cada CCAA.

5.Estas instituciones, además de coordinar las unidades de soporte territorial, deberán crear sólidos servicios especializados de asesoramiento e infraestructuras y plataformas de soporte a la investigación en AP, y deberán establecer las políticas de relación y participación con otras instituciones e infraestructuras de investigación cooperativa existentes, tanto nacionales como internacionales.

Actualmente, en España existen distintos modelos de organización. Ahora bien, los que se adaptan mejor a las características definidas anteriormente son los 3 modelos siguientes:

Organizaciones mixtas: gerencia de atención primaria con una fundación. La forma más frecuente como se ha iniciado la mayoría de experiencias de investigación en AP ha sido a partir de la creación de unidades de investigación dentro de las gerencias de AP, 
normalmente acompañadas de las unidades docentes. Sin embargo, éstas han tenido que evolucionar y trabajar conjuntamente con una fundación para darles el soporte necesario a la gestión económica de los proyectos y a la gestión y contratación de recursos humanos implicados en investigación. Este modelo, que combina la gerencia de AP (o gerencia única) y una fundación, es el modelo que existe en Baleares (Gerencia de AP de Mallorca con la FISIB), en Málaga (Gerencia de AP de Málaga con la Fundación IMABIS), en Vizcaya (Osakidetza de Vizcaya con la Fundación BIO Eusko Fundazioa), en algunas gerencias de Galicia, o en Salamanca donde el Centro de Salud La Alamedilla lidera la investigación de AP con una fundación privada. Normalmente, este modelo se adapta muy bien a las características y necesidades de la AP. Sin embargo, son organizaciones relativamente pequeñas, que no gestionan grandes infraestructuras o plataformas de investigación y están poco especializadas en la gestión de la investigación. Dentro de este modelo también encaja el de Cuenca, pero en este caso la relación con la Gerencia de AP se hace con la Universidad, la cual le da soporte en gestión económica y de recursos humanos además de asesoramiento y soporte en infraestructuras. En general, en este modelo sería interesante poder superar las barreras provinciales y poder coordinar territorios más amplios.

Programas integrados en organizaciones especializadas de investigación no específicas de AP: en las comunidades autónomas en las que se ha creado un instituto de investigación que coordina toda la investigación sanitaria de la comunidad se están creando programas específicos de investigación en $A P$, muchas veces a partir de la integración de unidades de investigación de las gerencias de AP. Esto sucede, a menudo, cuando se crean los institutos de investigación sanitaria acreditados por el Instituto de Salud Carlos III. Son ejemplos de este modelo el Programa de AP del Instituto Aragonés de Ciencias de la Salud (IACS), o el caso del Instituto de Investigación Sanitaria de Santiago de Compostela, integrando grupos de investigación de AP. Son institutos altamente especializados en investi- gación, aunque a veces se adaptan poco a las necesidades de la AP. Es muy importante que en estos institutos, con grupos muy potentes y con una alta producción, existan verdaderas políticas de apoyo a la investigación en AP, si no, esta seguirá siendo la "cenicienta" de la investigación. Por otra parte, es importante que la AP no se fragmente en territorios relativamente pequeños como pueden ser las zonas de influencia de un hospital.

Organizaciones de investigación específicas de AP: fundaciones o institutos creados específicamente para investigar en AP, con estructuras especializadas de soporte, coordinados íntimamente con las entidades de provisión de servicios de AP y con las otras organizaciones de investigación de la comunidad. El Instituto de Investigación en Atención Primaria Jordi Gol (IDIAP Jordi Gol) es el ejemplo más claro, coordinando toda la investigación de la AP en Catalunya. Se adaptan muy bien a las características definidas anteriormente.

Es importante, por tanto, que en cada comunidad autónoma, aprovechando las experiencias, oportunidades y características locales, se consoliden estructuras que permitan promocionar y desarrollar una investigación de excelencia en AP. A su vez, es fundamental que estas organizaciones se coordinen entre ellas para potenciar estructuras cooperativas a nivel nacional e internacional. Para ello es esencial vertebrar mejor las políticas locales, autonómicas y del Instituto de Salud Carlos III para trabajar conjuntamente en la misma dirección, complementarse y mejorar la actual organización de la investigación en AP. 


\section{BIBLIOGRAFÍA}

1. Hummers-Pradier E, et al. Research agenda for General Practice/Family Medicine and Primary Health Care in Europe. European General Practice Research Network (EGPRN), Maastricht 2009. Disponible en: http://www.egprn.org/images/Research\%20Agenda\%20for\%20General\%20Practice_ Family\%20Medicine.pdf (consultado 10/01/2011).

2. Mant D, Del Mar C, Glasziou P, Knottnerus A, Wallace $P$, van Weel C. The state of primary-care research. Lancet. 2004; 364(9438):1004-6.

3. Violan C, Bolibar B. Investigación biomédica. Papel de la atención primaria. Med Clin (Barc) 2006; 126:614-5.

4. Bolíbar Ribas B, Grandes Odriozola G, Llobera Canaves J, Bellón Saameño JA. La red de investigación en actividades preventivas y promoción de la salud: un reto para la atención primaria. Aten Primaria. 2007; 39(12):633-5.

Bonaventura Bolíbar Ribas Director Científico del IDIAP Jordi Gol.

Coordinador de la redIAPP (Red de Investigación en Actividades Preventivas en Atención Primaria)

Concepción Violan Fors Director Gerente del IDIAP Jordi Gol 